\title{
Imaging and Energy-Loss Characteristics for in vitro TEM Analysis of Nanoparticles
}

\author{
K.L. Klein, ${ }^{*}$ N. de Jonge, $* * * * *$ and I.M. Anderson* \\ *Surface and Microanalysis Science Division, NIST, Gaithersburg MD 20899-8371 \\ **Molecular Physiology and Biophysics, Vanderbilt U. Medical Center, Nashville TN 37232-0615 \\ ***Materials Science and Technology Division, Oak Ridge Natl. Lab., Oak Ridge TN 37831-6064
}

With the vast potential applications of nanomaterials, there is rising concern over the still undetermined toxicity and fate of engineered nanoparticles upon their release into our bodies and our surroundings. Toxicology studies in the literature, for the most part, consist of highly conflicting, incomparable results due to many uncertainties in the critical material properties. There is an urgent need for a technique that combines the ability to characterize functionalized nanostructures in their relevant aqueous (in vitro) environment with the high spatial resolution necessary to resolve individual nanostructures. The development of such a technique would provide unique and pertinent insights into the behavior of functionalized nanostructures, and would open the door to dynamic studies that could not otherwise be addressed.

This research seeks to establish methods for the characterization of nanoparticles in solution by conventional transmission electron microscopy (TEM) using a customized liquid flow cell specimen holder [1]. This paper presents an overview of the approach and some preliminary results toward characterizing the bright-field imaging of $\mathrm{Au}$ nanoparticles through a fluid layer of several micrometers thickness. Data were acquired from a liquid cell with a buffer solution contained between two ultra-thin SiN windows using a using a $300 \mathrm{kV}$ FEG TEM equipped with an imaging energy filter. A specialized holder interfaces with the specimen cell to provide fluid circulation. In order to characterize the flow cell thickness $(t)$ and intrinsic properties, 10-nm-diameter $\mathrm{Au}$ nanoparticles (NIST reference material 8011) were deposited onto the top and bottom exterior membrane surfaces. The thickness of the fluid layer was determined by parallax shift $(p)$ over a tilt angle of $\pm \gamma$, where $t=p / 2 \sin \gamma$ [2]. A parallax shift of $187 \mathrm{~nm}$ over $\pm 1^{\circ}$ tilt, implying a fluid thickness of $\approx 5.3 \mu \mathrm{m}$, is shown in Fig. 1. Interestingly, it was found that local displacement of the fluid occurred upon irradiation above a threshold dose, thus allowing the imaging characteristics and inelastic scattering response to be collected and compared from the same flow cell through liquid water and water vapor (essentially corresponding to the filled and unfilled cell), as shown in Fig. 2. While the energy-loss profile for the unfilled cell is typical of that for electron transparent specimens with thickness a fraction of an inelastic mean free path, the zero-loss intensity in the liquid-filled cell is negligible due to multiple inelastic scattering through the water, and the intensity maximum is $\approx$ $540 \mathrm{eV}$. Furthermore, while high resolution and good signal-to-noise ratio can be obtained from the $\mathrm{Au}$ nanoparticles on the unfilled cell, with the introduction of a fluid layer, it becomes advantageous to acquire filtered images integrated over the broad energy-loss range [3].

\section{References}

[1] N. de Jonge et al., PNAS 106 (2009) 2159.

[2] L. Reimer and H. Kohl, Transmission Electron Microscopy, Springer, New York, 2008.

[3] We are grateful to Protochips Inc. and for support from Vanderbilt University Medical Center (NJ) and the National Research Council Postdoctoral Research Associateship Program (KLK). 


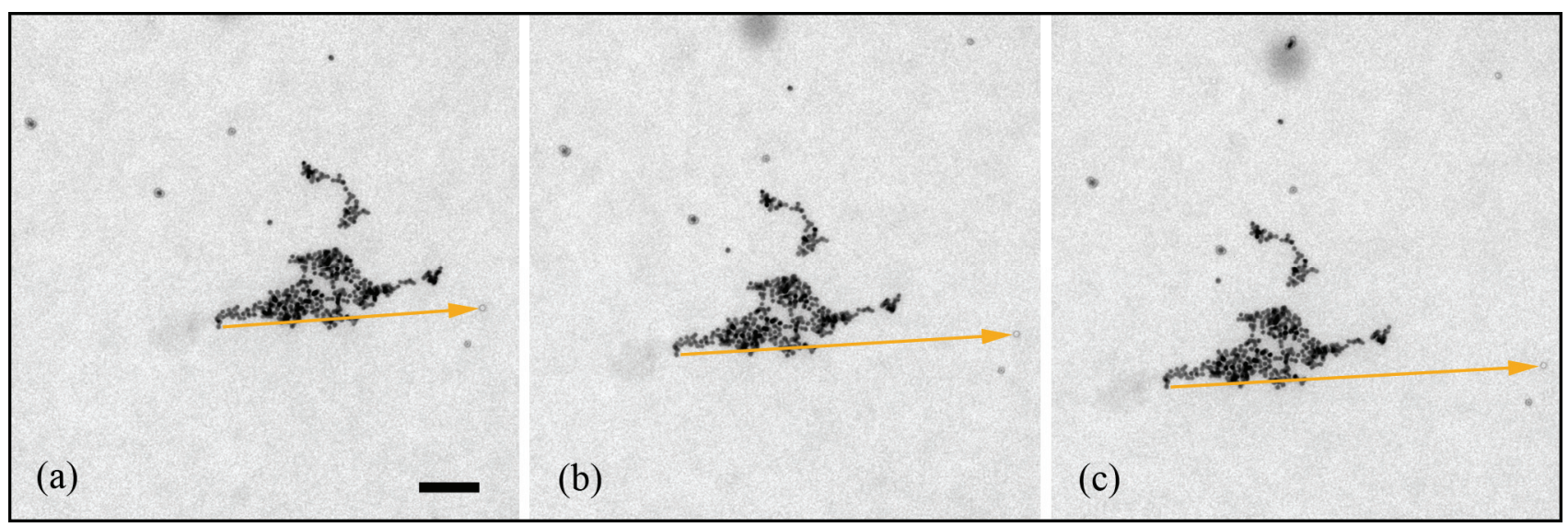

FIG 1. Parallax shift of $10 \mathrm{~nm}$ Au nanoparticles through tilts (a) $-1^{\circ}$ to (b) $0^{\circ}$ to (c) $+1^{\circ}$, used to determine the flow cell thickness $t$. Scale bar is $100 \mathrm{~nm}$.

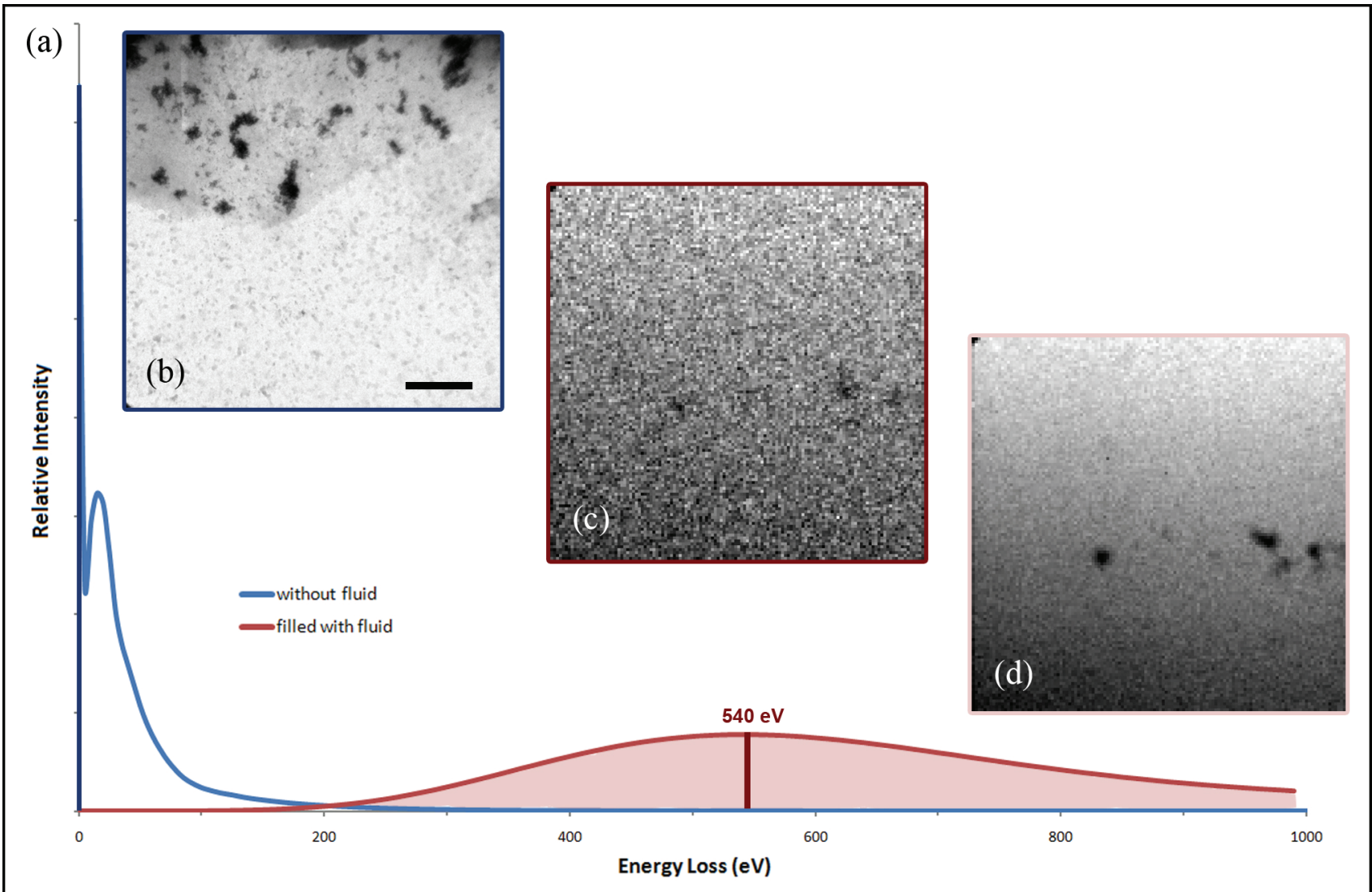

FIG 2. Energy-loss profiles and corresponding images extracted from EFTEM spectral images (SIs) acquired from a flow cell with and without fluid. 128x128 pixel SIs were acquired with $1 \mathrm{~s}$ per frame, a $20 \mathrm{eV}$ slit and $10 \mathrm{eV}$ step size. All images are at the same magnification; the scale bar is $500 \mathrm{~nm}$. (a) The presence of the fluid dramatically changes the energy-loss profile. The intensity axis is scaled so that the two profiles have the same integrated intensity. (b) Zero-loss image acquired from the unfilled cell shows aggregates of Au nanoparticles on the flow cell membrane. (c) An individual filtered image slice at $540 \mathrm{eV}$ through $\approx 5.3 \mu \mathrm{m}$ of fluid exhibits low signal-to-noise ratio, so that particles on the membrane are barely distinguishable. (d) Particle aggregates are clearly visible through the fluid-filled cell with the SI integrated from 0 to $1000 \mathrm{eV}$. 